\title{
Preoperative steroid treatment does not improve markers of inflammation after cardiac surgery in neonates: Results from a randomized trial
}

\author{
Eric M. Graham, MD, ${ }^{\text {a }}$ Andrew M. Atz, MD, ${ }^{a}$ Kimberly E. McHugh, MD, ${ }^{a}$ Ryan J. Butts, MD, ${ }^{a}$ \\ Nathaniel L. Baker, MS, ${ }^{b}$ Robert E. Stroud, MS, ${ }^{c}$ Scott T. Reeves, MD, ${ }^{d}$ Scott M. Bradley, MD, \\ Francis X. McGowan, Jr, MD, ${ }^{\mathrm{d}}$ and Francis G. Spinale, MD, $\mathrm{PhD}^{\mathrm{e}}$
}

\begin{abstract}
Objective: Neonatal cardiac surgery requiring cardiopulmonary bypass results in a heightened inflammatory response. Perioperative glucocorticoid administration is commonly used in an attempt to reduce the inflammatory cascade, although characterization of the cytokine response to steroids in neonatal cardiac surgery remains elusive because of highly variable approaches in administration. This randomized trial was designed to prospectively evaluate the effect of specific glucocorticoid dosing protocols on inflammatory markers in neonatal cardiac surgery requiring cardiopulmonary bypass.
\end{abstract}

\begin{abstract}
Methods: Neonates scheduled for cardiac surgery were randomly assigned to receive either 2-dose ( 8 hours preoperatively and operatively, $\mathrm{n}=36$ ) or single-dose (operatively, $\mathrm{n}=32$ ) methylprednisolone at $30 \mathrm{mg} / \mathrm{kg}$ per dose in a prospective double-blind trial. The primary outcome was the effect of these steroid regimens on markers of inflammation. Secondary analyses evaluated the association of specific cytokine profiles with postoperative clinical outcomes.
\end{abstract}

\begin{abstract}
Results: Patient demographics, perioperative variables, and preoperative indices of inflammation were similar between the single- and 2-dose groups. Preoperative cytokine response after the 2-dose methylprednisolone protocol was consistent with an anti-inflammatory effect, although this did not persist into the postoperative period. Premedication baseline levels of interleukin-6, interleukin-8, interleukin-10, and tumor necrosis factor $\alpha$ were predictive of postoperative intensive care unit and hospital length of stay. Only interleukin- 8 demonstrated a postoperative response associated with duration of intensive care unit and hospital stay.
\end{abstract}

Conclusions: The addition of a preoperative dose of methylprednisolone to a standard intraoperative methylprednisolone dose does not improve markers of inflammation after neonatal cardiac surgery. The routine administration of preoperative glucocorticoids in neonatal cardiac surgery should be reconsidered. ( $\mathrm{J}$ Thorac Cardiovasc Surg 2014;147:902-8)

\section{Earn CME credits at}

http://cme.ctsnetjournals.org
From the Division of Cardiology, ${ }^{\text {a }}$ Department of Pediatrics, Department of Public Health Sciences, ${ }^{\mathrm{b}}$ Division of Cardiothoracic Surgery, ${ }^{\mathrm{c}}$ Department of Surgery, and Anesthesiology and Perioperative Medicine, ${ }^{\mathrm{d}}$ Medical University of South Carolina, Charleston; and Departments of Surgery and Cell Biology and Anatomy, USC School of Medicine, Columbia, SC.

This work was supported by a Career Development Award from the American College of Cardiology Foundation/Pfizer Scholarship (to E.M.G.), NIH grants HL057952 and HL059165 (to F.G.S.), and the Research Service of the Department of Veterans Affairs.

Disclosures: Authors have nothing to disclose with regard to commercial support.

Dr Graham had full access to all the data in the study and takes responsibility for the integrity of the data and the accuracy of the data analysis.

Received for publication March 2, 2013; revisions received April 12, 2013; accepted for publication June 14, 2013; available ahead of print July 17, 2013.

Address for reprints: Eric M. Graham, MD, Medical University of South Carolina, 165 Ashley Ave, MSC 915, Charleston, SC 29425 (E-mail: grahamem@musc. edu).

0022-5223/\$36.00

Copyright (c) 2014 by The American Association for Thoracic Surgery

http://dx.doi.org/10.1016/j.jtcvs.2013.06.010
Cardiopulmonary bypass $(\mathrm{CPB})$ is a complex pathophysiologic environment in which exposure to nonphysiologic surfaces in the pump circuit, hemolysis, and ischemiareperfusion injury combine to initiate a complex cascade that includes proinflammatory cytokines, anti-inflammatory cytokines, and products of neutrophil activation. ${ }^{1-3}$ The inflammatory consequences from CPB culminate in a systemic inflammatory response syndrome. The systemic inflammatory response syndrome is further exacerbated in neonates because of morbidity factors of smaller patient size, greater hemodilution, hypothermia, and longer CPB times, resulting in a postoperative recovery period that is longer and more complex than similar operations performed in older infants and children. ${ }^{4-6}$

Clinical appreciation of this post-CPB inflammatory response has resulted in several interventions directed toward its reduction. ${ }^{7-9}$ Glucocorticoid administration is among the most common method to attenuate the clinical and biochemical features of the post-CPB inflammatory response, although the technique of steroid administration in neonatal cardiac surgery is highly variable. ${ }^{8,9}$ To further 


\section{Abbreviations and Acronyms \\ $\mathrm{CPB}=$ cardiopulmonary bypass \\ ICU = intensive care unit \\ IL = interleukin \\ LCOS $=$ low cardiac output syndrome \\ $\mathrm{MP}=$ methylprednisolone \\ $\mathrm{TNF}=$ tumor necrosis factor}

confound matters, the few studies investigating clinical outcomes associated with the use of corticosteroids in children requiring CPB yield contradictory results. ${ }^{10-13}$

Given the intertwined and redundant inflammatory cascade and the highly variable clinical practices in neonatal preoperative and intraoperative care, characterizing the anti-inflammatory response of steroids in neonatal cardiac surgery has remained elusive. Accordingly, this randomized trial was designed to prospectively evaluate the effect of uniform and specific glucocorticoid dosing protocols on inflammatory markers in the context of neonatal cardiac surgery requiring $\mathrm{CPB}$.

\section{METHODS}

\section{Patient Selection, Enrollment, and Randomization}

The study was approved by the Institutional Review Board, and informed written consent was obtained from the parent or legal guardian in accordance with all policies and regulations regarding obtaining informed consent of a minor. The study was part of a clinical outcomes trial, and the design details and clinical outcomes have been published (ClinicalTrials.gov Identifier: NCT00934843). ${ }^{10}$ In brief, inclusion criteria consisted of all inpatient neonates (aged $\leq 30$ days) scheduled to undergo a cardiac operation requiring $\mathrm{CPB}$. Exclusion criteria included prematurity (defined as $\leq 36$ weeks' postmenstrual age) at the time of surgery, treatment with steroids in the 2 weeks before surgery, suspected infection that would contraindicate steroid use (eg, herpes), known hypersensitivity to methylprednisolone (MP), or other contraindication to steroid therapy (eg, gastrointestinal tract bleeding). Subjects were randomly assigned to either preoperative placebo (approximately 8 hours preoperatively) and intraoperative MP at $30 \mathrm{mg} / \mathrm{kg}$ of body weight (single-dose group) or preoperative and intraoperative MP (2-dose group) within strata, according to planned corrective or palliative operation. All investigation and clinical site personnel were blinded to the treatment allocation until the close of the study.

\section{Outcome Variables}

The primary outcome was markers of inflammation after administration of specific glucocorticoid dosing protocols. The correspondence of cytokine profiles with clinical outcomes, such as low cardiac output syndrome (LCOS) and intensive care unit (ICU) and hospital stays, was investigated in secondary analyses. The presence of LCOS was defined by the clinicial signs and symptoms of low cardiac output (eg, tachycardia, oliguria, cold extremities, and cardiac arrest) that require 1 or more of the following interventions: mechanical circulatory support, the escalation of exisiting pharmacologic circulatory support to more than $100 \%$ over baseline, or the inititation of new pharmacologic circulatory support. ${ }^{14}$ The determination of LCOS was made by 2 independent reviewers (E.M.G., A.M.A.) and then agreed on between both reviewers before unblinding. Markers of inflammation were compared between treatment groups, which included the plasma levels of the proinflammatory cytokines, interleukin (IL)-2,
IL-6, IL-8, tumor necrosis factor $\alpha$ (TNF- $\alpha)$, and interferon- $\gamma$, and the anti-inflammatory cytokine, IL-10. The rationale for the selection of these cytokines was to survey both proinflammatory and anti-inflammatory markers. Samples were collected at 6 time points: (1) "baseline," before preoperative placebo/MP treatment (approximately 8 hours preoperatively), (2) immediately before skin incision, (3) on completion of modified ultrafiltration, and at (4) 4 hours, (5) 12 hours, and (6) 24 hours postoperatively. Whole blood samples of $1 \mathrm{~mL}$ were collected in ethylenediaminetetraacetic acid tubes at each of the 6 time points. Plasma was isolated by centrifugation, decanted into aliquots, and stored at $-80^{\circ} \mathrm{C}$ until processed for immunoassays.

Plasma levels of cytokines were determined by multiplex suspension array using commercially available and validated kits (R\&D Systems, Minneapolis, Minn). ${ }^{3}$ Plasma values were corrected for hemodilution using hematocrit values. All samples were batched and run simultaenously to avoid potential laboratory assay variance. Patients enrolled in the parent study were included in this analysis if they had an adequate volume of plasma at each of the 6 time points to analyze all inflammatory markers. Plasma-free hemoglobin, as a measurement of hemolysis, was obtained postoperatively on arrival to the ICU.

\section{Statistical Analysis}

Standard descriptive statistics were used to summarize the general demographic and clinical data. Continuous demographic characteristics are listed as means and associated SDs and were compared between groups using a $t$-test. Categorical characteristics are expressed as the number and percentage of subjects and were compared using a normal Pearson $\chi^{2}$ test statistic. Because of their skewed distributions, baseline measures of inflammatory markers are expressed as the median and interquartile range and are compared across steroid groups using a Wilcoxon rank-sum test statistic. Preoperative indices of inflammation were measured at "baseline" before administration of the preoperative steroids (or placebo) and again before surgery (preincision). To appraise the pharmacologic effect of preoperative MP steroid dose on cytokine levels, an analysis of variance model was used, fitting the preoperative steroid levels while controlling for the baseline premedication levels.

To assess the effect of steroid treatment on the cytokine profiles after surgery, a linear mixed-effect model was used. Restricted maximum likelihood methods were used to estimate the fixed effects and variance components in the presence of unbalanced data. ${ }^{15}$ Baseline cytokine values (premed), randomized treatment assignment, and aprotinin use status were used as covariates in a regression model, and estimate statements were used to construct group-level mean tests across steroid treatment groups at each time point. All cytokine post hoc pairwise comparison results are adjusted using the Bonferonni method. Because of the highly skewed and nonnormal distributions of the markers of inflammation, the values were $\log _{10}$ transformed before model analysis.

To test whether baseline or peak cytokine levels are associated with clinical postoperative outcomes (ICU and hospital lengths of stay), regression models were developed that adjusted for study design variables (randomized steroid treatment group and aprotinin administration). Independent associations were present between sex and surgery type (corrective vs palliative) with both ICU and hospital lengths of stay. Thus, all regression models were additionally adjusted for both characteristics. To test whether increases in baseline or peak cytokine levels are associated with higher odds to develop postoperative LCOS, similarly adjusted logistic regression models were developed. Statistical analyses were performed with SAS, version 9.2 (SAS Institute, Inc, Cary, NC).

\section{RESULTS}

\section{Preoperative Demographics and Intraoperative Variables}

Enrollment and outcomes have been previously reported. ${ }^{10}$ Briefly, 97 patients were screened for enrollment, and 78 met all inclusion/exclusion criteria and were randomized. 
Two subjects did not receive interventions using $\mathrm{CPB}$ and were excluded postrandomization. The remaining 76 randomized patients were assigned to the preoperative placebo and intraoperative MP (single dose, $\mathrm{n}=37$ ) or preoperative and intraoperative MP ( 2 dose, $n=39)$ protocol. Of the 76 subjects in the parent study, 68 had inflammatory markers measured at all 6 time points and were included in this analysis (single dose, $\mathrm{n}=32 ; 2$ dose, $\mathrm{n}=36$ ). Patient demographics, cardiac diagnoses, clinical characteristics, and operative and immediate postoperative procedures were similar between groups (Table 1). Similarly, overall CPB times, cross-clamp times, the use of deep hypothermic circulatory arrest, and immediate postoperative procedures were similar between the groups and have been previously reported. ${ }^{10}$ Mean plasmafree hemoglobin levels on arrival to the ICU were $71 \pm 36$ $\mathrm{mg} / \mathrm{dL}$ in the single-dose group and $75 \pm 40 \mathrm{mg} / \mathrm{dL}$ in the 2-dose group $(P=.69)$.

\section{Preoperative Indices of Inflammation}

Before study drug administration, baseline cytokine levels were similar between groups (Table 1). Immediately before skin incision, the proinflammatory cytokine IL- 6 and TNF$\alpha$ values decreased by $40 \%$ or more $(P<.01)$ (Figure $1, A$ and $D$ ) and the anti-inflammatory cytokine IL-10 value increased nearly 5 -fold in the 2 -dose group $(P<.01)$ (Figure 1,C), consistent with a pharmacologic effect of the preoperative MP dose. A pharmacologic response to preoperative MP occurred in most patients in the 2-dose group, as evident by $92 \%(33 / 36)$ having a decline in IL-6 levels between these preoperative time points. Although absolute IL- 8 values before skin incision were similar between groups, when adjusted for baseline values, there was a significant effect of preoperative MP administration $(P<.01)$ (Figure $1, B)$. IL-2 and interferon- $\gamma$ were low at baseline (Table 1) and not affected by preoperative MP administration.

\section{Postoperative Indices of Inflammation}

Dynamic changes in cytokine levels occurred in the postoperative period, indicative of altered inflammatory response in neonatal cardiac surgery. Robust increases in the proinflammatory responses of IL- 6 and IL- 8 occurred in the early postoperative period in both groups $(P<.01)$. These increases were similar between the single- and 2-dose groups (Figure 1). Immediately after modified ultrafiltration, IL-6 levels in the single-dose group remained numerically, but not significantly, higher than in the

TABLE 1. Preoperative demographics and clinical characteristics in neonatal patients undergoing cardiac surgery, randomized to either a singleor 2-dose methylprednisolone strategy

\begin{tabular}{|c|c|c|c|}
\hline Baseline demographic and clinical characteristics & MP single dose $(n=32)$ & MP 2 dose $(n=36)$ & $P$ value \\
\hline \multicolumn{4}{|l|}{ Demographics } \\
\hline Gestational age at birth, wk & $38.4 \pm 1.4$ & $38.9 \pm 1.2$ & .21 \\
\hline Gestational age at surgery, wk & $39.5 \pm 1.4$ & $40.1 \pm 1.5$ & .08 \\
\hline Age at surgery, $d$ & $7.7 \pm 4.3$ & $8.7 \pm 5.3$ & .27 \\
\hline Aged $\leq 7 \mathrm{~d}$, no. $(\%)$ & $25(78)$ & $22(61)$ & .13 \\
\hline Weight at surgery, $\mathrm{kg}$ & $3.1 \pm 0.5$ & $3.3 \pm 0.5$ & .37 \\
\hline Prenatal diagnosis, no. $(\%)$ & $14(44)$ & $18(50)$ & .61 \\
\hline Male sex, no. $(\%)$ & $16(50)$ & $20(56)$ & .65 \\
\hline \multicolumn{4}{|l|}{ Markers of inflammation, $\mathrm{pg} / \mathrm{mL}$} \\
\hline IL-2 & $1.1(0.9-1.3)$ & $1.2(1.0-1.3)$ & .15 \\
\hline IL-6 & $6.7(3.5-9.4)$ & $8.8(3.7-21.2)$ & .33 \\
\hline IL-8 & $22(17-42)$ & $31(19-47)$ & .25 \\
\hline IL-10 & $1.2(0.8-2.1)$ & $1.4(0.9-2.4)$ & .45 \\
\hline TNF- $\alpha$ & $6.8(4.1-8.7)$ & $5.9(4.2-8.2)$ & .56 \\
\hline IFN- $\gamma$ & $0.10(0.09-0.12)$ & $0.11(0.10-0.13)$ & .19 \\
\hline \multicolumn{4}{|l|}{ Diagnosis } \\
\hline Corrective Procedure & $17(53)$ & $19(53)$ & .97 \\
\hline Aortic arch hypoplasia with VSD & 3 & 5 & \\
\hline Tetralogy of Fallot & 2 & 2 & \\
\hline Transposition of the great arteries & 9 & 9 & \\
\hline Truncus arteriosis & 1 & 2 & \\
\hline Other biventricular repair & 2 & 1 & \\
\hline Palliative Procedure & $15(47)$ & $17(47)$ & \\
\hline Hypoplastic left heart syndrome & 5 & 8 & \\
\hline Other single-ventricle lesions & 8 & 7 & \\
\hline Pulmonary atresia with intact ventricular septum & 0 & 2 & \\
\hline Tetralogy of Fallot with pulmonary atresia & 2 & 0 & \\
\hline
\end{tabular}

Data are reported as mean $\pm \mathrm{SD}$, number (\%), or median (interquartile range), as appropriate. $M P$, Methylprednisolone; $I L$, interleukin; $T N F$, tumor necrosis factor; $I F N$, interferon; VSD, ventricular septal defect. 

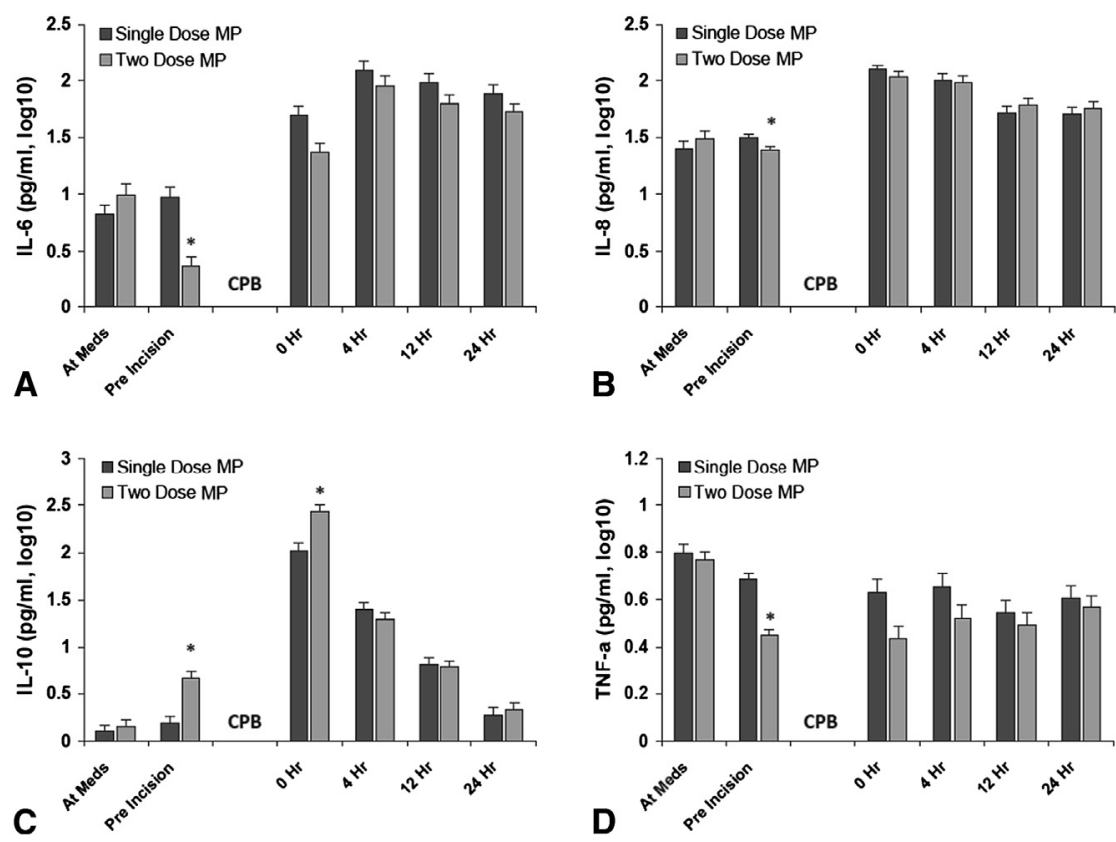

FIGURE 1. Cytokine profile at baseline (at $M e d s)$ and response to methylprednisolone $(M P)$ or placebo before cardiopulmonary bypass $(C P B ;$ preincision) and after CPB $(0,4,12$, and 24 hours after surgery) compared between treatment groups for logarithm-transformed values of (A) interleukin (IL)-6, (B) IL-8, (C) IL-10, and (D) tumor necrosis factor $(T N F)-\alpha .{ }^{*} P<.05$ (Bonferonni adjusted). $H r$, Hour; $M P$, methylprednisolone.

2-dose group $(P=.054)$. The anti-inflammatory molecule, IL-10, also had a significant increase in the postoperative period in both groups $(P<.01)$ and was higher in the 2-dose group immediately after modified ultrafiltration $(P=.001)$ (Figure $1, C)$, but was similar to the singledose group at all other postoperative time points. The pleotropic molecule, TNF- $\alpha$, was higher in the single-dose group immediately before CBP but failed to maintain this difference for the duration of the postoperative follow-up.

\section{Preoperative Indices of Inflammation and Outcomes}

Baseline indices of a heightened inflammatory state were associated with clinical postoperative outcomes of interest. Of the 68 study participants, 29 (43\%) experienced LCOS. For the entire cohort, mean ICU length of stay was 11.3 days (SD, 16.5 days) and hospital stay was 23.5 days (SD, 21.1 days), with no difference in these clinical outcomes between treatment groups. Higher levels of IL-6, IL-8, and IL-10 at baseline were significantly associated with increased postoperative ICU and hospital lengths of stay (all $P<.04$ ) (Table 2). Increased levels of TNF- $\alpha$ at baseline were modestly associated with ICU $(P=.057)$ and hospital $(P=.044)$ lengths of stay. None of the baseline cytokine measures was associated with LCOS after surgery (all $P>.15$ ).

\section{Postoperative Indices of Inflammation and Outcomes}

Despite the association of baseline levels of cytokines with clinical outcomes, a similar association for most postoperative values was not statistically significant. The postoperative response of the proinflammatory cytokine, IL-8, was predictive of ICU and hospital stays $(\beta=.33$ and $\beta=.26$, respectively; $P<.001$ ) (Table 2). None of the other postoperative measured cytokines was predictive of outcomes. In addition, no peak cytokine response was predictive of LCOS.

\section{DISCUSSION}

This prospective, randomized study using 2 different MP dosing strategies yielded a balanced set of patients in each treatment arm and, thereby, provided control for several potentially confounding variables. The unique findings are 3 -fold. First, although the 2-dose MP protocol reduced preoperative proinflammatory cytokines and increased anti-inflammatory cytokine levels, consistent with an antiinflammatory effect, this effect did not persist into the postoperative period. Second, premedication baseline levels of IL-6, IL-8, IL-10, and TNF- $\alpha$ were associated with increased postoperative ICU and hospital lengths of stay. Third, although the baseline levels of several cytokines were predictive of postoperative outcomes, only the postoperative response of the proinflammatory cytokine, IL-8, was associated with an increased duration of ICU and hospital stays. None of the other cytokines measured was associated with LCOS or ICU or hospital stays. The results from this randomized MP dosing protocol indicate that preoperative glucocorticoid treatment does not improve early postoperative markers of inflammation in neonatal cardiac surgery when compared with a single intraoperative dose. These 
TABLE 2. Association between baseline and peak measures of inflammation with postoperative outcomes

\begin{tabular}{|c|c|c|c|c|c|c|}
\hline \multirow[b]{3}{*}{ Measures of inflammation } & \multicolumn{6}{|c|}{ Postoperative outcomes } \\
\hline & \multicolumn{2}{|c|}{ ICU length of stay } & \multicolumn{2}{|c|}{ Hospital length of stay } & \multicolumn{2}{|c|}{ Low cardiac output syndrome } \\
\hline & $\beta \pm \mathbf{S E}$ & $P$ value & $\beta \pm \mathbf{S E}$ & $P$ value & OR $(95 \%$ CI $)$ & $P$ value \\
\hline \multicolumn{7}{|l|}{ Baseline levels } \\
\hline $\log _{10}$ IL-6 & $0.14 \pm 0.06$ & .039 & $0.18 \pm 0.05$ & $<.001$ & $0.49(0.18-1.34)$ & .162 \\
\hline $\log _{10}$ IL-8 & $0.28 \pm 0.09$ & .002 & $0.19 \pm 0.07$ & .012 & $1.54(0.43-5.52)$ & .510 \\
\hline $\log _{10}$ IL-10 & $0.23 \pm 0.09$ & .017 & $0.20 \pm 0.08$ & .013 & $0.55(0.14-2.17)$ & .395 \\
\hline $\log _{10}$ TNF- $\alpha$ & $0.33 \pm 0.17$ & .057 & $0.29 \pm 0.14$ & .044 & $3.81(0.33-43.8)$ & .293 \\
\hline \multicolumn{7}{|l|}{ Postoperative levels } \\
\hline $\log _{10}$ IL-6 & $0.08 \pm 0.09$ & .364 & $0.06 \pm 0.08$ & .414 & $1.15(0.33-4.04)$ & .822 \\
\hline $\log _{10}$ IL-8 & $0.33 \pm 0.09$ & $<.001$ & $0.26 \pm 0.08$ & $<.001$ & $2.39(0.61-9.38)$ & .213 \\
\hline $\log _{10}$ IL-10 & $0.02 \pm 0.07$ & .792 & $0.03 \pm 0.06$ & .585 & $1.24(0.47-3.29)$ & .664 \\
\hline $\log _{10}$ TNF- $\alpha$ & $0.11 \pm 0.12$ & .371 & $0.16 \pm 0.10$ & .119 & $0.61(0.12-3.26)$ & .564 \\
\hline
\end{tabular}

Preoperative levels of inflammation were measured before administration of study medication. Postoperative levels include the peak results measured after surgery. All analysis results shown are adjusted for study design variables (steroid treatment assignment and aprotinin administration), sex, and surgery type. Both ICU and hospital lengths of stay were highly skewed and were also $\log _{10}$ transformed before analysis. $\beta$ Estimates from mixed-effects linear models are shown as $\beta \pm$ SE, and logistic regression results are shown as $\mathrm{OR}(95 \% \mathrm{CI}) . I C U$, Intensive care unit; $O R$, odds ratio; $C I$, confidence interval; $I L$, interleukin; $T N F$, tumor necrosis factor.

observations suggest that a more critical evaluation of the widespread use of glucocorticoids in these high-risk cardiac surgery patients would be appropriate.

\section{Glucocorticoid Dosing Strategies and Inflammatory Mediators: Past Studies}

Glucocorticoids exert their anti-inflammatory effects through a complex series of both genomic and nongenomic effects. The classic genomic mechanism of glucocorticoids is activated via the cytosolic glucocorticoid receptor, resulting in both increased expression of anti-inflammatory regulatory proteins and decreased production of proinflammatory proteins. ${ }^{16}$ The modification of gene expression requires hours to days for clinical effects, founding the hypothesis for steroid administration 8 to 12 hours before CPB. ${ }^{17}$ Some rapid clinical effects occur too promptly to be explained by a genetic mode of action, and have been attributed to nongenomic mechanisms, including specific interaction with the cytosolic glucocorticoid receptor, nonspecific interactions with cellular membranes, and specific interactions with membrane-bound glucocorticoid receptors. ${ }^{18}$

Glucocorticoid administration has been associated with unclear benefits and potential detrimental effects in both adults and children undergoing cardiac surgery. Despite this, there is widespread use of perioperative glucocorticoid administration in pediatric cardiac surgery, with $80 \%$ to $97 \%$ of centers using some form of glucocorticoid therapy. ${ }^{8,9}$ Few controlled trials of glucocorticoids in the setting of CPB in pediatrics can be found. Bronicki and colleagues $^{19}$ randomized 29 children (mean age, 28 months) to receive dexamethasone $(1 \mathrm{mg} / \mathrm{kg}, \mathrm{n}=15)$ or placebo $(n=14) 1$ hour before CPB. Similar to our findings, both groups demonstrated a significant elevation in IL-6 after CPB. However, dexamethasone-treated patients had a blunted IL-6 response when compared with placebo.
The authors reported an increase in TNF- $\alpha 2$ minutes after $\mathrm{CPB}$ in the placebo group. It remained more than 3 times higher 10 minutes after protamine administration in the placebo group, but this did not reach statistical significance. At 24 hours postoperatively, the mean values were virtually identical. Complement component $\mathrm{C} 3 \mathrm{a}$ and absolute neutrophil count were not affected by dexamethasone.

Lindberg and colleagues ${ }^{20}$ randomized 40 patients weighing more than $10 \mathrm{~kg}$ (mean age, 52 months) to receive dexamethasone $(1 \mathrm{mg} / \mathrm{kg}, \mathrm{n}=20)$ or placebo $(\mathrm{n}=20)$ after the induction of anesthesia. C-reactive protein, as a marker of inflammation, was lower in the treatment group on the first postoperative day. In this cohort, we have previously reported similar levels of C-reactive protein at 36 hours postoperatively between the single- and 2-dose groups. ${ }^{10}$ Schroeder and colleagues ${ }^{21}$ randomized 29 children (mean age, 4 months) to MP $(30 \mathrm{mg} / \mathrm{kg}, \mathrm{n}=15)$ or placebo $(\mathrm{n}=14) 4$ hours before CPB. Similar to our study, MP $(30 \mathrm{mg} / \mathrm{kg}$ ) was administered in the pump prime in all patients. Serum IL-6 was lower immediately and 4 hours after CPB in the combined MP compared with the intraoperative-only group, but was equally elevated in both groups by 24 hours. The antiinflammatory cytokine, IL-10, was elevated in the combined MP group at the end of CPB and returned to baseline in both groups by 24 hours, similar to this study. Interestingly, they measured RNA from atrial samples before and after CPB. Both groups had an increased expression of inflammatory mediators after CPB; however, the combined steroid group had a statistically significant reduction in the expression of regulated on activation normal $\mathrm{T}$ cell expressed and secreted, monocyte chemoattractant protein-1, IL-6, and intercellular adhesion molecule-1. Patients receiving preoperative steroids also had lower mRNA expression for monocyte chemoattractant protein-1, IL-6, and intercellular adhesion molecule-1 at bypass initiation, before myocardial ischemia. 
In a comparison of dosing strategies, Varan and colleagues $^{22}$ randomized 30 children (mean age, 48 months) to receive either high-dose MP $(30 \mathrm{mg} / \mathrm{kg})$ or low-dose MP (2 mg/kg) before the onset of CPB. Consistent with the findings of this study, plasma levels of IL-6, IL-8, C-reactive protein, and polymorphonuclear leukocyte counts were all elevated postoperatively in both groups, without a significant difference between groups.

\section{Inflammatory Response and Clinical Outcomes}

Few studies in neonates and infants can be found demonstrating a relationship between inflammatory mediators and postoperative recovery. Consistent with this study, Alcaraz and colleagues ${ }^{6}$ investigated 8 neonates and 19 infants undergoing $\mathrm{CPB}$ and demonstrated significant increases in IL-6, IL-8, and TNF in both groups postoperatively. The magnitude of the inflammatory response was greater in the neonates, particularly for IL-8. Moreover, the IL-8 response correlated with pulmonary dysfunction and longer ICU stay. In contrast, IL-6 and TNF response was not associated with clinical outcomes. In a larger study by Allan and colleagues, ${ }^{4}$ the authors demonstrated in 93 infants, 9 months or younger, undergoing low-to-moderate complexity cardiac surgery serum concentrations of IL-6 and IL-8 immediately after CPB and at 24 hours postoperatively, these were associated with ICU length of stay in multivariate analysis. However, despite reaching statistical significance, the variability in outcomes accounted for by these inflammatory mediators was only of modest clinical significance ( $4 \%-9 \%$ variability of a median ICU stay of 3 days). Furthermore, despite demonstrating a heightened preoperative inflammatory state in the neonatal population, this did not correlate with postoperative markers of inflammation or clinical outcomes. The authors concluded that, in infants undergoing low-to-moderate complexity cardiac surgery, the contribution of inflammatory mediator production to postoperative morbidity is relatively limited.

In contrast, Appachi and colleagues ${ }^{23}$ demonstrated that neonates with hypoplastic left heart syndrome had higher preoperative and postoperative IL-6 levels than those with transposition of the great arteries, and postoperative IL-6 concentration was associated with mortality in neonates with hypoplastic left heart syndrome. This work suggests that the inflammatory response may have a greater significance in more complex operations. However, unique to the current study is the sole inclusion of neonates, resulting in the inclusion of complex corrective and palliative operations and the potential increased morbidity associated with them.

\section{Study Limitations}

Limitations of the current study include the lack of a completely placebo group. Therefore, it remains unclear whether the dosing strategies used were equally effective or neither was effective. Thus, recommendations for or against intraoperative MP cannot be made. Given the theoretical benefits and the widespread use of intraoperative glucocorticoids, a completely placebo arm was thought to be inapproriate during the design of this study. Considered in total, the past and present studies highlight the need for a recently initiated randomized controlled trial comparing intraoperative glucocorticoids with placebo in neonatal cardiac surgery (ClinicalTrials.gov identifier: NCT01579513). The results of our trial do not preclude the efficacy of other glucocorticoid regimens or the potential importance of other inflammatory pathways that were not directly examined in this study.

\section{CONCLUSIONS}

In conclusion, in the largest pediatric and only exclusively neonatal randomized trial comparing the efficacy of preoperative glucocorticoid therapy with intraoperative glucocorticoid therapy alone for neonates undergoing corrective and palliative cardiac operations requiring $\mathrm{CPB}$, our data do not provide support for the addition of a preoperative dose of MP to a standard intraoperative dose alone. Although preoperative dosing was associated with an improvement in preoperative inflammatory markers, this did not persist in the postoperative period. In light of these findings, the routine use of preoperative glucocorticoids in neonatal cardiac surgery needs to be reconsidered. Alternative therapies, not necessarly directed at anti-inflammatory mediators, are needed.

\section{References}

1. Tarnok A, Emmrich F. Immune consequences of pediatric and adult cardiovascular surgery: report of the 7th Leipzig workshop. Cytometry B Clin Cytom. 2003; 54:54-7.

2. Levy JH, Tanaka KA. Inflammatory response to cardiopulmonary bypass. Ann Thorac Surg. 2003;75:S715-20.

3. McQuinn TC, Deardorff RL, Mukherjee R, Taylor AG, Graham EM, Atz AM et al. Circulating matrix metalloproteinase levels after ventricular septal defect repair in infants. $J$ Thorac Cardiovasc Surg. 2010;140:1257-65.

4. Allan CK, Newburger JW, McGrath E, Elder J, Psoinos C, Laussen PC, et al. The relationship between inflammatory activation and clinical outcome after infant cardiopulmonary bypass. Anesth Analg. 2010;111:1244-51.

5. Alcaraz AJ, Sancho L, Manzano L, Esquivel F, Carrillo A, Prieto A, et al. Newborn patients exhibit an unusual pattern of interleukin 10 and interferon gamma serum levels in response to cardiac surgery. J Thorac Cardiovasc Surg. 2002;123: 451-8.

6. Alcaraz AJ, Manzano L, Sancho L, Vigil MD, Esquivel F, Maroto E, et al. Different proinflammatory cytokine serum pattern in neonate patients undergoing open heart surgery: relevance of IL-8. J Clin Immunol. 2005;25:238-45.

7. Graham EM, Atz AM, Gillis J, Desantis SM, Haney AL, Deardorff RL, et al. Differential effects of aprotinin and tranexamic acid on outcomes and cytokine profiles in neonates undergoing cardiac surgery. J Thorac Cardiovasc Surg. 2012; 143:1069-76.

8. Allen M, Sundararajan S, Pathan N, Burmester M, Macrae D. Anti-inflammatory modalities: their current use in pediatric cardiac surgery in the United Kingdom and Ireland. Pediatr Crit Care Med. 2009;10:341-5.

9. Checchia PA, Bronicki RA, Costello JM, Nelson DP. Steroid use before pediatric cardiac operations using cardiopulmonary bypass: an international survey of 36 centers. Pediatr Crit Care Med. 2005;6:441-4.

10. Graham EM, Atz AM, Butts RJ, Baker NL, Zyblewski SC, Deardorff RL, et al Standardized preoperative corticosteroid treatment in neonates undergoing 
cardiac surgery: results from a randomized trial. J Thorac Cardiovasc Surg. 2011;142:1523-9.

11. Pasquali SK, Hall M, Li JS, Peterson ED, Jaggers J, Lodge AJ, et al. Corticosteroids and outcome in children undergoing congenital heart surgery: analysis of the Pediatric Health Information Systems database. Circulation. 2010;122:2123-30.

12. Pasquali SK, Li JS, He X, Jacobs ML, O’Brien SM, Hall M, et al. Perioperative methylprednisolone and outcome in neonates undergoing heart surgery. Pediatrics. 2012;129:e385-91.

13. Clarizia NA, Manlhiot C, Schwartz SM, Sivarajan VB, Maratta R, Holtby HM, et al. Improved outcomes associated with intraoperative steroid use in highrisk pediatric cardiac surgery. Ann Thorac Surg. 2011;91:1222-7.

14. Hoffman TM, Wernovsky G, Atz AM, Kulik TJ, Nelson DP, Chang AC, et al. Efficacy and safety of milrinone in preventing low cardiac output syndrome in infants and children after corrective surgery for congenital heart disease. Circulation. 2003;107:996-1002.

15. Patterson HD, Thompson R. Recovery of inter-block information when block sizes are unequal. Biometrika. 1971;58:545-64.

16. Stahn C, Buttgereit F. Genomic and nongenomic effects of glucocorticoids. Nat Clin Pract Rheumatol. 2008;4:525-33.

17. Lodge AJ, Chai PJ, Daggett CW, Ungerleider RM, Jaggers J. Methylprednisolone reduces the inflammatory response to cardiopulmonary bypass in neonatal piglets: timing of dose is important. $J$ Thorac Cardiovasc Surg. 1999;117:515-22.

18. Buttgereit F, Scheffold A. Rapid glucocorticoid effects on immune cells. Steroids. 2002;67:529-34

19. Bronicki RA, Backer CL, Baden HP, Mavroudis C, Crawford SE, Green TP. Dexamethasone reduces the inflammatory response to cardiopulmonary bypass in children. Ann Thorac Surg. 2000;69:1490-5.

20. Lindberg L, Forsell C, Jogi P, Olsson AK. Effects of dexamethasone on clinical course, C-reactive protein, S100B protein and von Willebrand factor antigen after paediatric cardiac surgery. Br J Anaesth. 2003;90:728-32.

21. Schroeder VA, Pearl JM, Schwartz SM, Shanley TP, Manning PB, Nelson DP. Combined steroid treatment for congenital heart surgery improves oxygen delivery and reduces postbypass inflammatory mediator expression. Circulation. 2003; 107:2823-8.

22. Varan B, Tokel K, Mercan S, Donmez A, Aslamaci S. Systemic inflammatory response related to cardiopulmonary bypass and its modification by methyl prednisolone: high dose versus low dose. Pediatr Cardiol. 2002;23: 437-41.

23. Appachi E, Mossad E, Mee RB, Bokesch P. Perioperative serum interleukins in neonates with hypoplastic left-heart syndrome and transposition of the great arteries. J Cardiothorac Vasc Anesth. 2007;21:184-90. 